\title{
Tagungsberichte
}

\section{Der Weg in die Nachhaltigkeit}

\section{Berlin Conference on the Human Dimensions of Global Environmental Change: Governance for Industrial Transformation}

Berlin, 5.-6. Dezember 2003

\section{Thomas Höfer}

Dr. Thomas Höfer, Bundesinstitut für Risikobewertung, Thiellallee 88-92, D-14195 Berlin (thomas.hoefer@bfr.bund.de)

In Berlin fand die dritte Konferenz zu den Herausforderungen der globalen Umweltveränderungen für die menschliche Gesellschaft, insbesondere der Herausforderungen an die Umweltpolitik statt. Die Konferenzen in den Jahren 2001 und 2002 beschäftigten sich mit der Rolle des Nationalstaats bei der globalen Umweltveränderung und dem Wissensmanagement für den Übergang in die Nachhaltigkeit. Der aktuelle Kongress, von einem Team der Forschungsstelle für Umweltpolitik der Freien Universität Berlin um Klaus Jacob getragen, stand unter dem Leitgedanken 'Politische Gestaltung des Übergangs in die Nachhaltigkeit'.

Die Gestaltung der Umweltpolitik hat sich in den letzten Jahren erheblich gewandelt: Das Spektrum der direkt Beteiligten hat sich erweitert und die Autorität der nationalen Regierungen wird von nicht-staatlichen Ákteuren infrage gestellt. Die Instrumente der Umweltpolitik haben ihren Schwerpunkt vom obrigkeitsstaatlichen Ansatz ('command and control') zur gestalterischen Zusammenarbeit (z.B. über wirtschaftliche Anreize) verschoben. Die Relevanz der nationalen Ebene hat in der Umweltpolitik abgenommen. Internationale Verflechung bestimmt das Bild.

Die klassische Politik gegenüber Umweltproblemen, deren Beseitigung in 5-20 Jahren möglich ist, konzentriert sich auf die Säuberung der Umwelt am Ende der Produktkette ('end-of-pipe'). Sie ist in der Regel erfolgreich, greift in die innere Gestaltung der Industriegesellschaft wenig ein und läßt den Unternehmen ihre eigene Strategie. In den letzten 10-15 Jahren entstand eine innovationsorientierte Umweltpolitik, die umweltfreundliche Technik im Markt etablieren will. Hierzu dienen Verbrauchermotivation, Subventionen für nachhaltigere Technologien und steuerliche Belastungen umweltschädlicher Technik. Diese Politik greift in Industrie- und Standortstrukturen ein. Grüne Umweltpolitik geht noch einen Schritt weiter: Sie entwickelt eine ökologische Modernisierung besonders entscheidender Branchen für die Umweltverschmutzung, wie der Strom-, Grundstoff- und der Chemieindustrie, greift damit tief in Industrie- und Wirtschaftsstrukturen ein. Eine Politik der Umgestaltung zur Nachhaltigkeit muss einen noch größeren Rahmen als die bestehenden politischen Ansätze umfassen. Eine Umkehr der Entwicklung zur Klimaveränderung, des Verlustes der Artenvielfalt und der Habitate wird Jahrzehnte oder Jahrhunderte brauchen. Wie kann sie politisch gestaltet werden? Fünf parallele Workshops diskutierten Perspektiven aufbauend auf Ergebnissen aktueller Forschungen, z.B.:

Welche Art der politischen Steuerung, der regionalen Initiativen, der Einbindung nationaler Interessen in internationale Abkommen und Institutionen ist geeignet, den Übergang in eine nachhaltige Industriegestaltung umzusetzen? Hier wurden z.B. die beschränkte Effektivität sogenannter lokaler Agenda 21 Aktivitäten und die globalen Abkommen mit ihrem deutlichen Umsetzungsmangel diskutiert.

Wie kann der Übergangsprozess in die Nachhaltigkeit erfolgreich gestaltet werden und welche Interventionen durch Staat und Forschung können wirksam sein? Die Komplexität der verwobenen Ent- wicklungen stellt eine politische Steuerung vor schwer lösbare $\mathrm{He}-$ rausforderungen. Die Segmentierung nationaler Zuständigkeiten und Organisationen hemmt die Wende. Eingriffsspielräume sind oft zeitlich eng begrenzt und müssen daher frühzeitig erkannt werden.

Wie kann die Vision einer nachhaltigen Bewirtschaftung die Entwicklung und die politische Umsetzung unterstützen? Welche Rollen können die Beteiligten haben? Wichtiger Diskussionspunkt war. hier die Kraft von Visionen für gesellschaftliche Veränderungen. Studien zeigten, dass die Promotion unpopulärer Visionen ausserordentlich kostspielig ist und negative Visionen derzeit die Umweltpolitik bestimmen. Europäische Wissenschaftler studieren Möglichkeiten des Einsatzes von Szenarien der Nachhaltigkeit.

Die Naturwissenschaften bieten Abschätzungen der Risiken einer nicht-nachhaltigen Entwicklung. Alternativen können sie aber nur begrenzt entwickeln. Martin Jänicke von der Freien Universität Berlin stellte 'Verhandlungen im Schatten der Hierachie' dar, bei denen Naturwissenschaftlern in Behörden und staatlichen Forschungszentren eine entscheidende Rolle bei Vereinbarungen zwischen Regierung und Wirtschaft zukommt. Ihr fachlich kompetenter und überzeugender Beitrag wirkt oft entscheidend bei der Konsensfindung. Der technische Wandel stellt aber nur einen Aspekt der Perspektive dar. Es seien grundsätzliche sozio-kulturelle Veränderungen in den Bereichen Energie (Verbrauch und Erzeugung), Nahrung (Ernährung, Landwirtschaft), Städte (Transport und Wasser), Information und Kommunikation als auch Politikgestaltung notwendig. Kritisches Echo fand der Vortrag von Nicholas Ashford vom Massachusetts Institute of Technology, der aufgrund seiner Arbeiten eine kritische Bilanz der "nur scheinbar funktionierenden Marktkräfte" ableitete. Hierunter zählten die unzutreffende Berücksichtigung der Umwelt- und der Arbeits-, sowohl der Sozial- als auch der Gesundheitskosten. Er betrachtet die Beschäftigungsfrage und die Sozialkosten als die in Zukunft bestimmende Größe beim Erfolg jeder Nachhaltigkeitsstrategie.

Es wurden während der Konferenz Stimmen laut, die vor der üblichen Strategie der Einbindung und Beteiligung aller Beteiligter auf vielen Ebenen aller Sektoren warnten. Sie fordern hingegen die Identifikation konkreter Ziele und klarer Verantwortlichkeiten mit einer deutlichen Rolle des Nationalstaats in der internationalen Umweltpolitik. Die internationalen Erfolgschancen solcher "steuernden Umweltpolitik" blieben jedoch umstritten.

Kritik entstand an der Nutzung des Schlagworts vom Übergang in die Nachbaltigkeit (Transition to Sustainability) für andere Politikfelder ausserhalb der Umweltpolitik, wie die Einbeziehung von Vereinsleben oder Kriminalität als 'Nachhaltigkeitsindikatoren'. Die Forderung nach Nachhaltigkeit kann so bald zum beliebigen politischen Schlagwort verkommen und damit Gefahr laufen, wirkungslos zu werden.

Viele Vorträge sind im Internet eingestellt, um allen Interessenten weltweit einen schnellen Zugriff zu ermöglichen: http://www. fu-berlin.de/ffu/akumwelt/bc2003/download.htm 